\title{
Randomized controlled trials in neurosurgery: an observational analysis of trial discontinuation and publication outcome
}

\author{
Aimun A. B. Jamjoom, MRCS, ${ }^{1}$ Angus B. Gane, BMedSci, ${ }^{2}$ and Andreas K. Demetriades, FRCS(SN) ${ }^{1}$ \\ 'Division of Neurosurgery, Department of Clinical Neuroscience, Western General Hospital; and ${ }^{2}$ Medical School, The University \\ of Edinburgh, United Kingdom
}

OBJECTIVE This study aimed to determine the trial discontinuation and publication rate of randomized controlled trials (RCTs) in neurosurgery.

METHODS Trials registered from 2000 to 2012 were identified on the website clinicaltrials.gov using a range of key words related to neurosurgery. Any trials that were actively recruiting or had unknown status were excluded. Included trials were assessed for whether they were discontinued early on the clinicaltrials.gov database; this included trials identified as withdrawn, suspended, or terminated in the database. For included trials, a range of parameters was identified including the subspecialty, primary country, study start date, type of intervention, number of centers, and funding status. Subsequently, a systematic search for published peer-reviewed articles was undertaken. For trials that were discontinued early or were found to be unpublished, principal investigators were sent a querying email.

RESULTS Sixty-four neurosurgical trials fulfilled our inclusion criteria. Of these $64,26.6 \%$ were discontinued early, with slow or insufficient recruitment cited as the major reason (57\%). Of the 47 completed trials, $14(30 \%)$ remained unpublished. Discontinued trials showed a statistically significant higher chance of remaining unpublished (88\%) compared with completed trials $(p=0.0002)$. Industry-funded trials had a higher discontinuation rate $(31 \%)$ compared with non-industryfunded trials $(23 \%)$, but this result did not reach significance $(p=0.57)$. Reporting of primary outcome measures was complete in $20(61 \%)$ of 33 trials. For secondary outcome measures, complete reporting occurred in only $11(33.3 \%)$ of 33.

CONCLUSIONS More than a fifth (26.6\%) of neurosurgical RCTs are discontinued early and almost a third of those that are completed remain unpublished. This result highlights significant waste of financial resources and clinical data.

https://thejns.org/doi/abs/10.3171/2016.8.JNS16765

KEY WORDS randomized controlled trial; trial discontinuation; publication rate; bibliometrics; diagnostic technique

$\mathrm{E}$ VIDENCE-BASED medicine relies on high-quality research to determine whether medical interventions are of benefit to patients. Randomized controlled trials (RCTs) provide the strongest evidence on the effectiveness of medical interventions. ${ }^{3}$ However, RCTs can only shape the evidence landscape through efficient trial completion and publication of results in the medical literature. Early trial discontinuation and nonpublication pose major issues for health care research and patient care due to the significant waste of resources surrounding uncompleted trials. In addition, unpublished data can distort and bias the literature and lead to underinformed decisions on medical interventions that can even lead to patient harm. ${ }^{4}$

A recent study by Chapman and colleagues aimed to assess the discontinuation and nonpublication rate of surgical RCTs. ${ }^{6}$ They found that from 395 surgical trials there was a discontinuation rate of $21 \%$ and a nonpublication rate of $34 \%$. However, that study only assessed 10 neurosurgical trials, which does not provide a robust assessment of the subspecialty's trial landscape. Neurosurgery, in particular, suffers from the difficulties in trial design that plague surgical RCTs, including the study of low-caseload diseases in which slow recruitment or small sample size may fail to result in well-matched groups following randomization. ${ }^{9}$ The problem of small sample sizes in neurosurgery is well known and was reported as "endemic" in the text Guiding Neurosurgery by Evidence (2006). ${ }^{17}$ Another issue facing neurosurgical trialists is increased risk

ABBREVIATIONS CONSORT = Consolidated Standards of Reporting Trials; IF = impact factor; $\mathrm{PI}$ = principal investigator; $\mathrm{RCT}=$ randomized controlled trial SUBMITTED March 25, 2016. ACCEPTED August 4, 2016.

INCLUDE WHEN CITING Published online November 25, 2016; DOI: 10.3171/2016.8.JNS16765. 
of patient dropout from studies that require long follow-up times. ${ }^{17}$ Alongside the logistical difficulties, neurosurgical RCTs also face ethical hurdles, such as difficulty obtaining consent from patients with impaired consciousness. While sham operations are considered by many to be an important control component used in neurosurgical RCTs, they are also a controversial area, as has been demonstrated by opposition to sham operations in the field of Parkinson's disease research. ${ }^{7}$ Due to these difficulties, we believed it was important to gain a greater understanding of the discontinuation and publication rates of neurosurgery trials.

The introduction of the trial registry clinicaltrials.org has helped provide an open access repository of registered trials and create more transparency concerning clinical trial data. Much effort has recently focused on trying to identify factors associated with early discontinuation and nonpublication of clinical trials, including the role of industry participation. ${ }^{8}$ Coupled with this effort, initiatives such as the Alltrials campaign (www.alltrials.net) seek to decrease the loss of data from RCTs and provide a framework by which we can acquire better evidence on clinical interventions while reducing resource wastage. ${ }^{4}$ Another major influence on the field has been made by the Consolidated Standards of Reporting Trials (CONSORT) Group who have produced minimum reporting standards for RCTs. ${ }^{2}$ This study aims to systematically review registered RCTs in neurosurgery and quantify the rates of trial discontinuation and publication.

\section{Methods}

A neurosurgical RCT was defined as any Phase 3 or 4 trial that assessed a procedure involving an incision to the head or spine and/or had a neurosurgeon as its principal investigator (PI). A list of key words to identify neurosurgical trials were devised using PubReMiner and informal literature searches to obtain MESH terms. The following list of key words were used to probe the clinicaltrials.gov database: "neurosurgery" OR "craniotomy" OR "discectomy" OR "laminectomy" OR "shunt" OR "external ventricular drain" OR "epilepsy surgery" OR "vagus nerve stimulator" OR "functional neurosurgery" OR "aneurysm clipping" OR "aneurysm surgery" OR "glioblastoma" OR "meningioma" OR "brain metastasis" OR "skull base" OR "deep brain stimulation."

Within our search parameters, a "first received" date range of January 1, 2000, to December 31, 2012, was stipulated, which allowed for completed trials to be published. This date range applied to when clinicaltrials.gov received the trial information rather than the trial start date. Trials identified as completed, withdrawn, suspended, or terminated were included within the study. A completed trial was defined as a clinical study that ended as planned and participants were no longer being treated or examined. Any trials that were actively recruiting or had unknown status were excluded. Following screening for status, 2 independent reviewers (A.B.G. and A.A.B.J.) determined whether trials were an RCT design and whether it fulfilled our criteria for a neurosurgical trial. Any disagreement was settled with discussion. For included trials a range of parameters were identified, including the neurosurgi- cal subspecialty, primary country, study start date, type of intervention (procedure, device, drug, other), number of centers (single or multicenter trial), and funding status (industry or nonindustry funded).

All included studies were then assessed for whether they had been published in a peer-reviewed journal. Clinicaltrials.gov trial profiles were assessed for published results and the trial and PI names were searched in PubMed. Trials that were found to have been published had further data points extracted from the publication, including: date of publication, 2014 journal impact factor (IF), and completeness of trial outcome measurements compared with those defined on the trial registration page at clinicaltrials. gov. For trials that were discontinued or unpublished, trial PIs were contacted to ascertain the reasons behind this status. All PIs were also contacted to ascertain approximate costs for running the included trials.

\section{Statistical Analysis}

Two main end points for the trials were defined: discontinuation and nonpublication. Discontinuation was defined as any trial that stopped early and this included trials identified on clinicaltrials.gov as "withdrawn," "terminated," or "suspended." Nonpublication was defined as any completed trial that did not have a published research article in a peer-reviewed journal. Figure generation and statistical analysis were achieved using Microsoft Office Excel and GraphPad Prism 6. Two-tailed chi-square tests were used to compare the different groups. A p value $\leq 0.05$ was considered statistically significant.

\section{Results}

A total of 723 trials were identified during the primary search phase using the predefined key words. From these trials, 307 trials were excluded because they were either actively recruiting or had unknown status. Of the remaining 416 trials, 64 met our definition of a neurosurgical RCT (Table 1). Figure 1 demonstrates the search process and reasons for trial exclusion. Among the included studies, 29 $(45 \%)$ were industry funded. The subspecialties included were spinal (27\%), functional and epilepsy (19\%), neurooncology (17\%), anesthesia and perioperative care (14\%), CSF (9\%), neurovascular (9\%), and neurotrauma (5\%). The studies assessed devices in 39\% of cases, followed by drugs in $33 \%$ and procedures in $28 \%$. The US was the primary country in more than half $(53 \%)$ of the trials, followed by France (8\%) and Canada (6\%).

\section{Discontinued Trials}

Of the included trials, 17 were terminated early, giving our cohort of trials a discontinuation rate of $26.6 \%$. Of the 17 terminated trials, $14(82 \%)$ provided a reason for discontinuation either on the clinicaltrials.gov website or by email from the trial PI. The main cause (57\%) of early termination was slow or insufficient patient recruitment. Table 2 highlights the other major reasons for early termination of the clinical trials. Of the discontinued trials, 2 were published in peer-reviewed articles, giving the cohort a nonpublication rate of $88 \%$. A further 3 trials made their results available through clinicaltrials.gov. 


\begin{tabular}{|c|c|c|c|c|c|c|c|}
\hline $\begin{array}{l}\text { Study } \\
\text { Title }\end{array}$ & Subspecialty & Intervention & Funding & Center & $\begin{array}{l}\text { Start } \\
\text { Date } \\
(\mathrm{Mo}-\mathrm{Yr})\end{array}$ & $\begin{array}{l}\text { End } \\
\text { Date } \\
(\mathrm{Mo-Yr})\end{array}$ & Status \\
\hline $\begin{array}{l}\text { Surgery vs stereotactic radiosurgery in the treatment of } \\
\text { single brain metastasis: a randomized trial }\end{array}$ & Neurooncology & Procedure & Nonindustry & Single & Jan-98 & Dec-05 & Completed \\
\hline Hypothermia during intracranial aneurysm surgery trial & Neurovascular & Procedure & Nonindustry & Single & Feb-00 & Unknown & Completed \\
\hline Surgical vs nonsurgical treatment for spinal stenosis & Spinal & Procedure & Nonindustry & Single & Sep-00 & Dec-07 & Completed \\
\hline COGNIShunt system for Alzheimer's disease & CSF & Device & Industry & Single & Jan-01 & Oct-04 & Completed \\
\hline Dexanabinol in severe traumatic brain injury & Neurotrauma & Drug & Industry & Single & Jan-01 & Sep-04 & Completed \\
\hline $\begin{array}{l}\text { STN-stimulation versus best medical treatment in ad- } \\
\text { vanced PD }\end{array}$ & $\begin{array}{l}\text { Functional \& } \\
\text { epilepsy }\end{array}$ & Procedure & Nonindustry & Multi & Jan-01 & Jan-05 & Completed \\
\hline Performance of the hedrocel cervical fusion device & Spinal & Device & Industry & Multi & Dec-01 & Jan-09 & Discontinued \\
\hline $\begin{array}{l}\text { Radiosurgery with or without whole-brain radiation therapy } \\
\text { in treating patients with brain metastases }\end{array}$ & Neurooncology & Procedure & Nonindustry & Multi & Dec-01 & Oct-14 & Completed \\
\hline $\begin{array}{l}\text { Decompressive craniectomy in malignant middle cerebral } \\
\text { artery infarcts }\end{array}$ & Neurovascular & Procedure & Nonindustry & Single & Dec-01 & May-16 & Discontinued \\
\hline rhBMP-2/CRM/CD HORIZON spinal system pivotal study & Spinal & Device & Industry & Multi & Mar-02 & Feb-10 & Completed \\
\hline $\begin{array}{l}\text { Phase I deep brain stimulation (DBS) vs best medical } \\
\text { therapy (BMT) trial }\end{array}$ & $\begin{array}{c}\text { Functional \& } \\
\text { epilepsy }\end{array}$ & Device & Nonindustry & Multi & Apr-02 & Oct-08 & Completed \\
\hline $\begin{array}{l}\text { Phase II subthalamic nucleus (STN) vs globus pallidus } \\
\text { (GPi) trial }\end{array}$ & $\begin{array}{c}\text { Functional \& } \\
\text { epilepsy }\end{array}$ & Device & Industry & Multi & Apr-02 & Apr-09 & Completed \\
\hline $\begin{array}{l}\text { A trial comparing radiosurgery with surgery for solitary } \\
\text { brain metastases }\end{array}$ & Neurooncology & Procedure & Nonindustry & Single & Dec-02 & May-09 & Completed \\
\hline A clinical study of the Dynesys spinal system & Spinal & Device & Industry & Multi & Mar-03 & Dec-15 & Discontinued \\
\hline Duragen vs Duraguard in Chiari surgery & CSF & Drug & Nonindustry & Single & Apr-03 & Apr-10 & Completed \\
\hline MAVERICK total disc replacement - pivotal study & Spinal & Device & Industry & Multi & Apr-03 & Nov-10 & Completed \\
\hline $\begin{array}{l}\text { Economic medical evaluation of treatment of the neuro- } \\
\text { pathic pain rebel by cortical stimulation }\end{array}$ & $\begin{array}{c}\text { Functional \& } \\
\text { epilepsy }\end{array}$ & Device & Nonindustry & Multi & Aug-03 & Dec-07 & Completed \\
\hline Surgery vs radiosurgery to treat metastatic brain tumors & Neurooncology & Procedure & Nonindustry & Single & Dec-03 & Nov-05 & Completed \\
\hline $\begin{array}{l}\text { The PRECISE trial: study of IL13-PE38QQR compared to } \\
\text { GLIADEL wafer in patients with recurrent glioblastoma } \\
\text { multiforme }\end{array}$ & Neurooncology & Drug & Industry & Multi & Feb-04 & Mar-07 & Completed \\
\hline $\begin{array}{l}\text { Impact of ventricular catheter used with antimicrobial } \\
\text { agents on patients with a ventricular catheter }\end{array}$ & CSF & Device & Nonindustry & Single & Apr-04 & Jun-09 & Completed \\
\hline PCM cervical disc system & Spinal & Device & Industry & Multi & Jan-05 & Aug-14 & Completed \\
\hline High light and low light dose PDT in glioma & Neurooncology & Drug & Nonindustry & Single & Mar-05 & Mar-06 & Completed \\
\hline $\begin{array}{l}\text { CONCEPT: crossover efficacy pain trial in motor cortex } \\
\text { stimulation for intractable neuropathic pain }\end{array}$ & $\begin{array}{l}\text { Functional \& } \\
\text { epilepsy }\end{array}$ & Device & Industry & Multi & Jun-05 & Mar-08 & Discontinued \\
\hline $\begin{array}{l}\text { Double-blind, multicenter study to assess the efficacy of } \\
\text { bilateral pallidal stimulation in patients with medically } \\
\text { refractory primary cervical dystonia }\end{array}$ & $\begin{array}{c}\text { Functional \& } \\
\text { epilepsy }\end{array}$ & Device & Industry & Multi & Jul-05 & Apr-08 & Completed \\
\hline $\begin{array}{l}\text { STIMEP: assessment of subthalamic nucleus stimulation } \\
\text { in drug resistant epilepsy }\end{array}$ & $\begin{array}{l}\text { Functional \& } \\
\text { epilepsy }\end{array}$ & Device & Nonindustry & Multi & Sep-05 & Mar-10 & Discontinued \\
\hline $\begin{array}{l}\text { Clinical efficacy of in-situ thrombolysis in case of intraven- } \\
\text { tricular haemorraghia by aneurysm rupture }\end{array}$ & Neurovascular & Procedure & Nonindustry & Single & Oct-05 & Dec-09 & Completed \\
\hline RNS system pivotal study & $\begin{array}{l}\text { Functional \& } \\
\text { epilepsy }\end{array}$ & Device & Industry & Multi & Dec-05 & May-11 & Completed \\
\hline $\begin{array}{l}\text { Study comparing best medical practice with or without } \\
\text { VNS therapy in pharmacoresistant partial epilepsy } \\
\text { patients }\end{array}$ & $\begin{array}{c}\text { Functional \& } \\
\text { epilepsy }\end{array}$ & Device & Industry & Multi & Feb-06 & Jul-08 & Discontinued \\
\hline $\begin{array}{l}\text { Effects of erythropoietin on cerebral vascular dysfunction } \\
\text { and anemia in traumatic brain injury }\end{array}$ & Neurotrauma & Drug & Nonindustry & Single & Apr-06 & Mar-13 & Completed \\
\hline $\begin{array}{l}\text { Discrete hypothermia in the management of traumatic } \\
\text { brain injury }\end{array}$ & Neurotrauma & Device & Nonindustry & Single & Jul-06 & Oct-08 & Completed \\
\hline
\end{tabular}




\begin{tabular}{|c|c|c|c|c|c|c|c|}
\hline $\begin{array}{l}\text { Study } \\
\text { Title }\end{array}$ & Subspecialty & Intervention & Funding & Center & $\begin{array}{l}\text { Start } \\
\text { Date } \\
\text { (Mo-Yr) }\end{array}$ & $\begin{array}{l}\text { End } \\
\text { Date } \\
(\text { Mo-Yr) }\end{array}$ & Status \\
\hline $\begin{array}{l}\text { Effect of NovoTTF-100A in recurrent glioblastoma multi- } \\
\text { forme (GBM) }\end{array}$ & Neurooncology & Device & Industry & Multi & Sep-06 & Jan-11 & Completed \\
\hline $\begin{array}{l}\text { Safety and effectiveness study of the TOPS system, a total } \\
\text { posterior arthroplasty implant designed to alleviate pain } \\
\text { resulting from moderate to severe lumbar stenosis }\end{array}$ & Spinal & Device & Industry & Multi & Sep-06 & Unknown & Completed \\
\hline Effect of Parecoxib on post-craniotomy pain & $\begin{array}{l}\text { Anesthesia \& } \\
\text { periop care }\end{array}$ & Drug & Nonindustry & Single & Sep-06 & Dec-08 & Completed \\
\hline $\begin{array}{l}\text { Herniectomy vs herniectomy with a spinal stabilization } \\
\text { system for the treatment of complex disc disease }\end{array}$ & Spinal & Device & Industry & Multi & Nov-06 & Jun-13 & Completed \\
\hline $\begin{array}{l}\text { Simvastatin in aneurysmal subarachnoid haemorrhage } \\
\text { (STASH) a multicentre randomised controlled clinical } \\
\text { trial }\end{array}$ & Neurovascular & Drug & Nonindustry & Multi & Jan-07 & Feb-14 & Completed \\
\hline $\begin{array}{l}\text { Randomized study of anular repair with the Xclose tissue } \\
\text { repair system }\end{array}$ & Spinal & Procedure & Industry & Multi & Mar-07 & Jan-12 & Completed \\
\hline $\begin{array}{l}\text { Neural stem cell preserving brain radiation therapy \& } \\
\text { stereotactic radiosurgery in patients with 1-6 brain } \\
\text { metastases }\end{array}$ & Neurooncology & Procedure & Nonindustry & Single & Mar-07 & Jun-09 & Discontinued \\
\hline Nimotuzumab in adults with glioblastoma multiforme & Neurooncology & Drug & Industry & Multi & Aug-07 & Jan-12 & Completed \\
\hline $\begin{array}{l}\text { Aprepitant with dexamethasone versus ondansetron with } \\
\text { dexamethasone for PONV prophylaxis in patients hav- } \\
\text { ing craniotomy }\end{array}$ & $\begin{array}{l}\text { Anesthesia \& } \\
\text { periop care }\end{array}$ & Drug & Industry & Single & Sep-07 & Jun-09 & Completed \\
\hline Preemptive analgesia for postlaminectomy & $\begin{array}{l}\text { Anesthesia \& } \\
\text { periop care }\end{array}$ & Drug & Nonindustry & Single & Sep-07 & Dec-07 & Completed \\
\hline Effects of X-STOP vs laminectomy study & Spinal & Device & Industry & Single & Nov-07 & Nov-11 & Discontinued \\
\hline $\begin{array}{l}\text { Clazosentan in reducing vasospasm-related morbidity and } \\
\text { all-cause mortality in adult patients with aneurysmal } \\
\text { subarachnoid hemorrhage treated by surgical clipping }\end{array}$ & Neurovascular & Drug & Industry & Multi & Nov-07 & Jul-10 & Completed \\
\hline The Tilburg vasospasm study & Neurovascular & Procedure & Nonindustry & Single & Dec-07 & Jun-09 & Discontinued \\
\hline Anesthesiological strategies in elective craniotomy & $\begin{array}{l}\text { Anesthesia \& } \\
\text { periop care }\end{array}$ & Drug & Nonindustry & Multi & Dec-07 & Dec-09 & Completed \\
\hline $\begin{array}{l}\text { Dexmedetomidine effects on microelectrode recording in } \\
\text { deep brain stimulation }\end{array}$ & $\begin{array}{c}\text { Functional \& } \\
\text { epilepsy }\end{array}$ & Drug & Nonindustry & Single & Jan-08 & Jan-12 & Discontinued \\
\hline $\begin{array}{l}\text { A multicenter study of hippocampal electrical stimulation } \\
\text { (HS) in mesial temporal lobe epilepsy }\end{array}$ & $\begin{array}{c}\text { Functional \& } \\
\text { epilepsy }\end{array}$ & Procedure & Nonindustry & Multi & Jan-08 & Mar-12 & Discontinued \\
\hline $\begin{array}{l}\text { Wallis interspinous dynamic stability system for lumbar } \\
\text { disc herniation: a prospective study }\end{array}$ & Spinal & Procedure & Nonindustry & Single & Jul-08 & Oct-12 & Completed \\
\hline Pre-lemniscal radiation deep brain stimulation for ET & $\begin{array}{c}\text { Functional \& } \\
\text { epilepsy }\end{array}$ & Procedure & Nonindustry & Single & Oct-08 & Unknown & Discontinued \\
\hline $\begin{array}{l}\text { Efficacy and safety of AP } 12009 \text { in patients with recur- } \\
\text { rent or refractory anaplastic astrocytoma or secondary } \\
\text { glioblastoma }\end{array}$ & Neurooncology & Drug & Industry & Multi & Dec-08 & Jun-12 & Discontinued \\
\hline $\begin{array}{l}\text { Comparison of propofol to precedex with propofol for } \\
\text { emergence and recovery in patients having craniotomy } \\
\text { surgery }\end{array}$ & $\begin{array}{l}\text { Anesthesia \& } \\
\text { periop care }\end{array}$ & Drug & Nonindustry & Single & Feb-09 & Feb-10 & Completed \\
\hline $\begin{array}{l}\text { Study of epidural steroid injection (ESI) vs minimally } \\
\text { invasive lumbar decompression (MILD) in patients with } \\
\text { symptomatic lumbar central canal stenosis }\end{array}$ & Spinal & Procedure & Industry & Single & Aug-09 & May-13 & Completed \\
\hline $\begin{array}{l}\text { Clinical trial to evaluate safety and efficacy of Hyalospine } \\
\text { in lumbar laminectomy or laminotomy }\end{array}$ & Spinal & Device & Nonindustry & Multi & Nov-09 & Nov-12 & Completed \\
\hline $\begin{array}{l}\text { Spinal cord stimulation with precision system vs reopera- } \\
\text { tion for failed back surgery syndrome }\end{array}$ & Spinal & Device & Industry & Multi & Feb-10 & May-13 & Discontinued \\
\hline
\end{tabular}


TABLE 1. Table summarizing included studies

\begin{tabular}{|c|c|c|c|c|c|c|c|}
\hline $\begin{array}{l}\text { Study } \\
\text { Title }\end{array}$ & Subspecialty & Intervention & Funding & Center & $\begin{array}{l}\text { Start } \\
\text { Date } \\
(\mathrm{Mo}-\mathrm{Yr})\end{array}$ & $\begin{array}{l}\text { End } \\
\text { Date } \\
(\text { Mo-Yr) }\end{array}$ & Status \\
\hline $\begin{array}{l}\text { Safety and effectiveness of the Adherus dural sealant sys- } \\
\text { tem when used as a dural sealant in cranial procedures }\end{array}$ & CSF & Device & Industry & Multi & Jun-10 & Jan-13 & Completed \\
\hline $\begin{array}{l}\text { A study to evaluate the safety and effectiveness of EVICEL } \\
\text { as an adjunct to sutured dural repair }\end{array}$ & CSF & Drug & Industry & Multi & Jul-10 & Oct-11 & Completed \\
\hline $\begin{array}{l}\text { Surgery and whole brain radiotherapy }(\mathrm{RT}) \text { vs whole brain } \\
\text { radiotherapy }(\mathrm{RT}) \text { and radiosurgery for } 1-3 \text { resectable } \\
\text { brain metastases }\end{array}$ & Neurooncology & Procedure & Nonindustry & Single & Jul-10 & Jul-10 & Discontinued \\
\hline TASALL - TachoSil against liquor leak & CSF & Procedure & Industry & Multi & Apr-11 & Jun-13 & Completed \\
\hline $\begin{array}{l}\text { Comparison of dexmedetomidin and remifentanil for the ef- } \\
\text { fect on airway reflex and hemodynamic response during } \\
\text { emergence in patients undergoing craniotomy }\end{array}$ & $\begin{array}{l}\text { Anesthesia \& } \\
\text { periop care }\end{array}$ & Drug & Nonindustry & Single & May-11 & Sep-12 & Completed \\
\hline $\begin{array}{l}\text { Evaluation of fusion rate of anterior cervical discectomy } \\
\text { and fusion (ACDF) using Cervios and Bonion }\end{array}$ & Spinal & Device & Nonindustry & Single & Nov-11 & Aug-13 & Completed \\
\hline $\begin{array}{l}\text { The PRIORi-T Trial - prospective randomized investigation } \\
\text { of radiofrequency targeted vertebral augmentation }\end{array}$ & Spinal & Device & Industry & Multi & Nov-11 & Unknown & Discontinued \\
\hline $\begin{array}{l}\text { Reduction bleeding in laminectomy with double doses of } \\
\text { tranexamic acid }\end{array}$ & Spinal & Drug & Nonindustry & Single & Jun-12 & Dec-13 & Completed \\
\hline $\begin{array}{l}\text { Diclofenac administered before skull operations reduces } \\
\text { the severity of headache after the intervention }\end{array}$ & $\begin{array}{l}\text { Anesthesia \& } \\
\text { periop care }\end{array}$ & Drug & Nonindustry & Single & Dec-12 & Apr-13 & Completed \\
\hline $\begin{array}{l}\text { Effects of perioperative pregabalin for post-craniotomy } \\
\text { pain }\end{array}$ & $\begin{array}{l}\text { Anesthesia \& } \\
\text { periop care }\end{array}$ & Drug & Industry & Single & Aug-13 & Unknown & Discontinued \\
\hline $\begin{array}{l}\text { Correlation of measured and calculated serum osmolality } \\
\text { during hyperosmolar drugs infusion in patients after } \\
\text { craniotomy }\end{array}$ & $\begin{array}{l}\text { Anesthesia \& } \\
\text { periop care }\end{array}$ & Drug & Nonindustry & Single & Jan-14 & Jun-14 & Completed \\
\hline
\end{tabular}

ET = essential tremor; PD = Parkinson's disease; PDT = photodynamic therapy; PONV = postoperative nausea and vomiting; RNS = responsive neurostimulation; STN $=$ subthalamic nucleus; VNS = vagus nerve stimulation.

The neurosurgical subspecialty with the highest discontinuation rate was functional and epilepsy neurosurgery $(50 \%)$ followed by neurovascular $(33 \%)$ and spinal $(29 \%$; Table 3). Trials focusing on drugs had a lower discontinu- ation rate (14\%) compared with trials focusing on devices (32\%) and procedures (33\%). Industry-funded trials had a nonsignificantly $(\mathrm{p}=0.57)$ higher termination rate $(31 \%)$ compared with nonindustry-funded trials $(23 \%)$. The

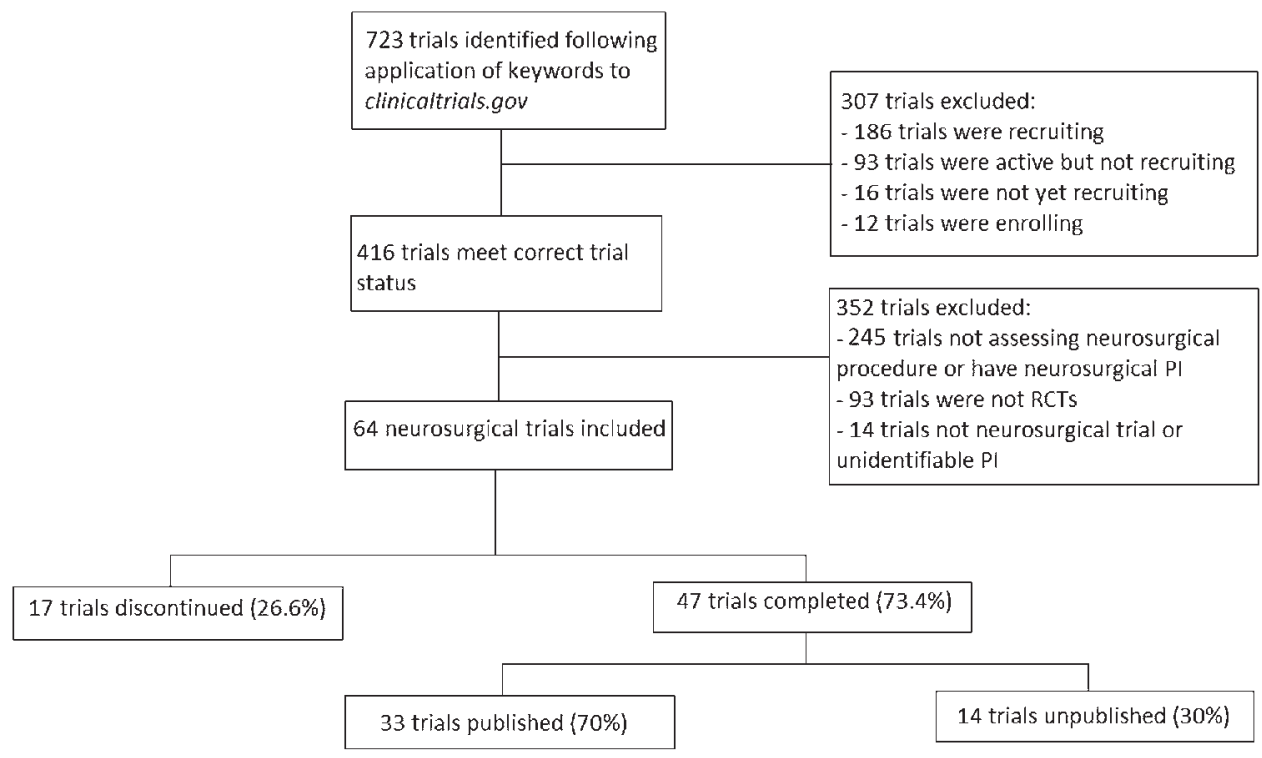

FIG. 1. Flow diagram highlighting trial inclusion and outcomes. 
TABLE 2. Data regarding the reasons for trial termination

\begin{tabular}{lc}
\hline \multicolumn{1}{c}{ Reason for Termination } & No. of Trials* $(\%)$ \\
\hline Slow or insufficient enrollment & $8(57)$ \\
\hline Administrative issues & $3(21)$ \\
\hline Procedural/technical challenges & $2(14)$ \\
\hline Patient preconceptions inhibited randomization & $1(7)$ \\
\hline New research priorities & $1(7)$ \\
\hline One arm evidently superior-unethical to continue & $1(7)$ \\
\hline
\end{tabular}

* There were 14 total trials included; 3 trials did not have information on the reason for discontinuation. Note that some trials cited multiple reasons for noncompletion and as a result have been coded multiple times.

number of involved centers also did not affect the trial discontinuation rate, with single-center trials having a nonsignificantly $(\mathrm{p}=0.82)$ higher discontinuation rate $(28 \%)$ compared with multicenter trials $(25 \%)$. Temporal changes of trial discontinuation rate demonstrated an increase from $16.7 \%$ in $2002-2005$ up to $33.3 \%$ in 2010-2014 (Fig. 2).

A total of 12 responses were received regarding trial costs. From these responses, 2 responders did not know their trial costs, 3 advised that their trials did not receive funding and could not provide a cost, and 7 provided an estimated cost. Of these 7 studies with cost data, 2 were discontinued early. The average cost across the 7 studies was $\$ 2,593,086$, with a range of $\$ 22,400-\$ 11,000,000$. The 2 discontinued trials had a cumulative cost of $\$ 314,400$.

\section{Completed Trials}

Of the 47 completed trials, we found 33 published research articles in peer-reviewed journals, giving the cohort a nonpublication rate of $30 \%$. Completed trials had a significantly lower nonpublication rate compared with discontinued trials $(88 \% ; \mathrm{p}=0.0002)$. Of the 14 unpublished tri-
TABLE 3. Details of the differing discontinuation rates based on a range of parameters

\begin{tabular}{lcc}
\hline \multicolumn{1}{c}{ Parameter } & Total & Discontinued (\%) \\
\hline Neurosurgical subspecialty & & \\
\hline Anesthesia \& periop care & 9 & $1(11)$ \\
\hline CSF & 6 & $0(0)$ \\
\hline Functional \& epilepsy & 12 & $6(50)$ \\
\hline Neurooncology & 11 & $3(27)$ \\
\hline Neurotrauma & 3 & $0(0)$ \\
\hline Neurovascular & 6 & $2(33)$ \\
\hline Spinal & 17 & $5(29)$ \\
\hline Intervention type & & \\
\hline Device & 25 & $8(32)$ \\
\hline Drug & 21 & $3(14)$ \\
\hline Procedure & 18 & $6(33)$ \\
\hline Funding status & & $9(31)$ \\
\hline Industry & 29 & $8(23)$ \\
\hline Nonindustry & 35 & $8(25)$ \\
\hline No. of centers & & $9(28)$ \\
\hline Multicenter & 32 & \\
\hline Single center & 32 & \\
\hline
\end{tabular}

als, 4 provided some data on the clinicaltrials.gov registry. Of the 3 PIs who responded to our email, 2 of their studies had not been published because of a failure to accrue sufficient numbers of participants. In 1 of these cases, the manuscript had been submitted for publication but rejected for this reason. A manuscript was in preparation for the final unpublished trial. Trials assessing CSF management $(50 \%)$ and spinal (42\%) and neurooncological (38\%) interventions had the highest nonpublication rates (Table 4).

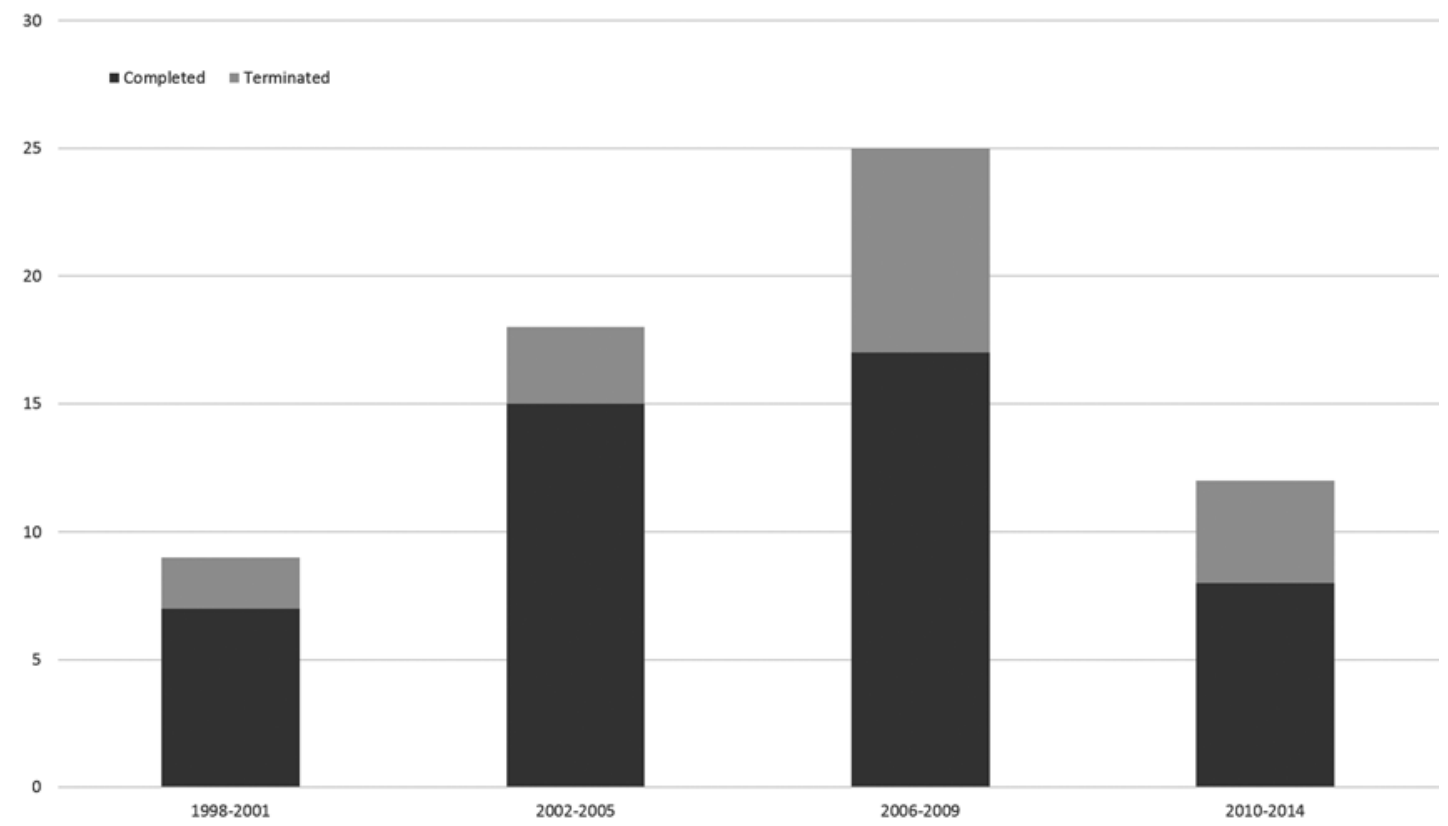

FIG. 2. Bar chart demonstrating temporal change in trial discontinuation from 1998 to 2014 . 
TABLE 4. Details on the differing nonpublication rates based on a range of parameters

\begin{tabular}{lcc}
\hline \multicolumn{1}{c}{ Parameter } & Completed & Nonpublished (\%) \\
\hline Neurosurgical subspecialty & & \\
\hline Anesthesia \& periop care & 8 & $2(25)$ \\
\hline CSF & 6 & $3(50)$ \\
\hline Functional \& epilepsy & 6 & $1(17)$ \\
\hline Neurooncology & 8 & $3(38)$ \\
\hline Neurotrauma & 3 & $0(0)$ \\
\hline Neurovascular & 4 & $0(0)$ \\
\hline Spinal & 12 & $5(42)$ \\
\hline Intervention type & & \\
\hline Device & 17 & $7(41)$ \\
\hline Drug & 18 & $3(17)$ \\
\hline Procedure & 12 & $4(33)$ \\
\hline Funding status & & \\
\hline Industry & 20 & $6(30)$ \\
\hline Nonindustry & 27 & \\
\hline No. of centers & & $8(30)$ \\
\hline Multicenter & 23 & $6(25)$ \\
\hline Single center & 24 &
\end{tabular}

Multicenter trials had a higher nonpublication rate at $35 \%$ compared with single-center trials (25\%), but this did not reach statistical significance $(\mathrm{p}=0.59)$. Industry- and nonindustry-funded trials had the same nonpublication rate of $30 \%(\mathrm{p}=0.98)$. Trials assessing devices had the highest nonpublication rate $(41 \%)$, followed by procedures $(33 \%)$ and drugs (17\%).

For the published articles, the median time from the study start date to publication was 7.6 years, with a range of 1.8-17.5 years. Articles were published in journals with a median 2014 IF of 4.9, with a range of no IF to 55.8. The study populations reported in the research articles ranged from 19 to 1147, with a median population of 169 patients. Primary outcome measures were completely reported in $61 \%$ of the trials while the reporting of secondary outcome measures was more scarce, with only $33.3 \%$ of the trials reporting these measures (Fig. 3). Data on outcome measure reporting in individual neurosurgical subspecialties is shown in Fig. 4. This figure highlights that neurosurgical trials focusing on anesthesia/perioperative care and functional operations had the best reporting record of registered primary and secondary outcome measures.

\section{Discussion}

This study demonstrated that more than one-fifth of neurosurgical RCTs are discontinued early and almost a third of those that are completed remain unpublished. These data highlight that neurosurgery suffers from the same waste of resources and ethical concerns surrounding trial discontinuation and nonpublication that have been described and discussed in the literature. ${ }^{10}$

To our knowledge, this study is the most comprehensive assessment of these issues within the neurosurgical literature. Two recent studies have examined the issue of surgical RCT discontinuation and publication outcome with differing methodologies and outcomes. Chapman and colleagues used clinicaltrials.gov as a registration platform and found a discontinuation rate of $21 \% .^{6}$ This study only evaluated 10 neurosurgical trials, however, which constituted only $3 \%$ of the studied cohort, limiting the generalizability of the results to the neurosurgical literature. Conversely, Rosenthal et al. used trial protocols approved by 6 ethics committees as the resource to follow up RCTs. ${ }^{18}$ The study found that $43 \%$ of the 127 surgical RCTs had been discontinued early. Rosenthal et al.'s study did not identify what proportion of the trials was neurosurgical. Comparing our study to the 2 above, we believe our methodologies

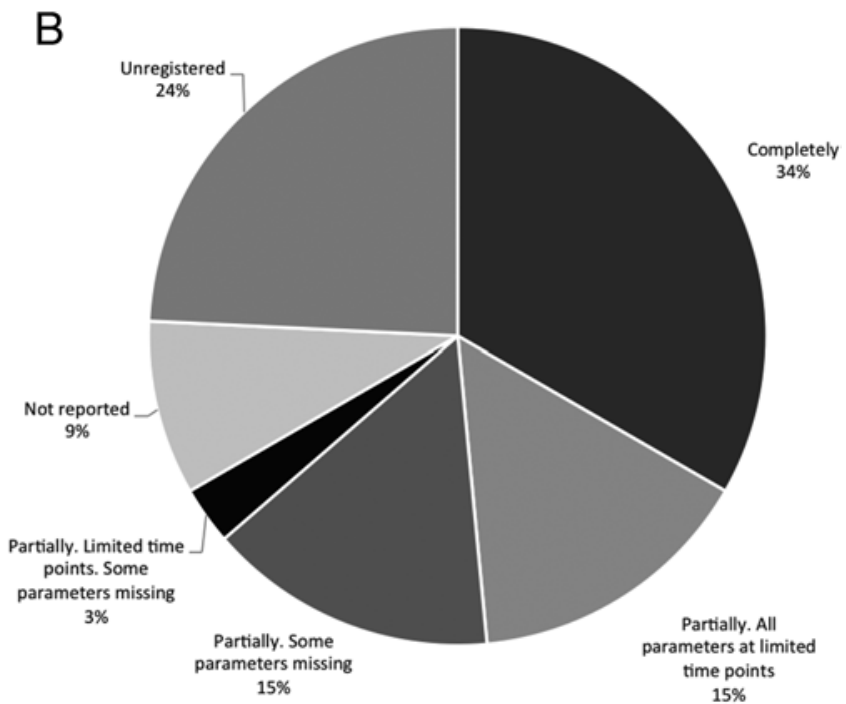

FIG. 3. Pie charts showing the reporting of (A) primary outcome measures and (B) secondary outcome measures for published trials. "Unregistered" means no outcome measures were uploaded to the clinicaltrials.gov database. 

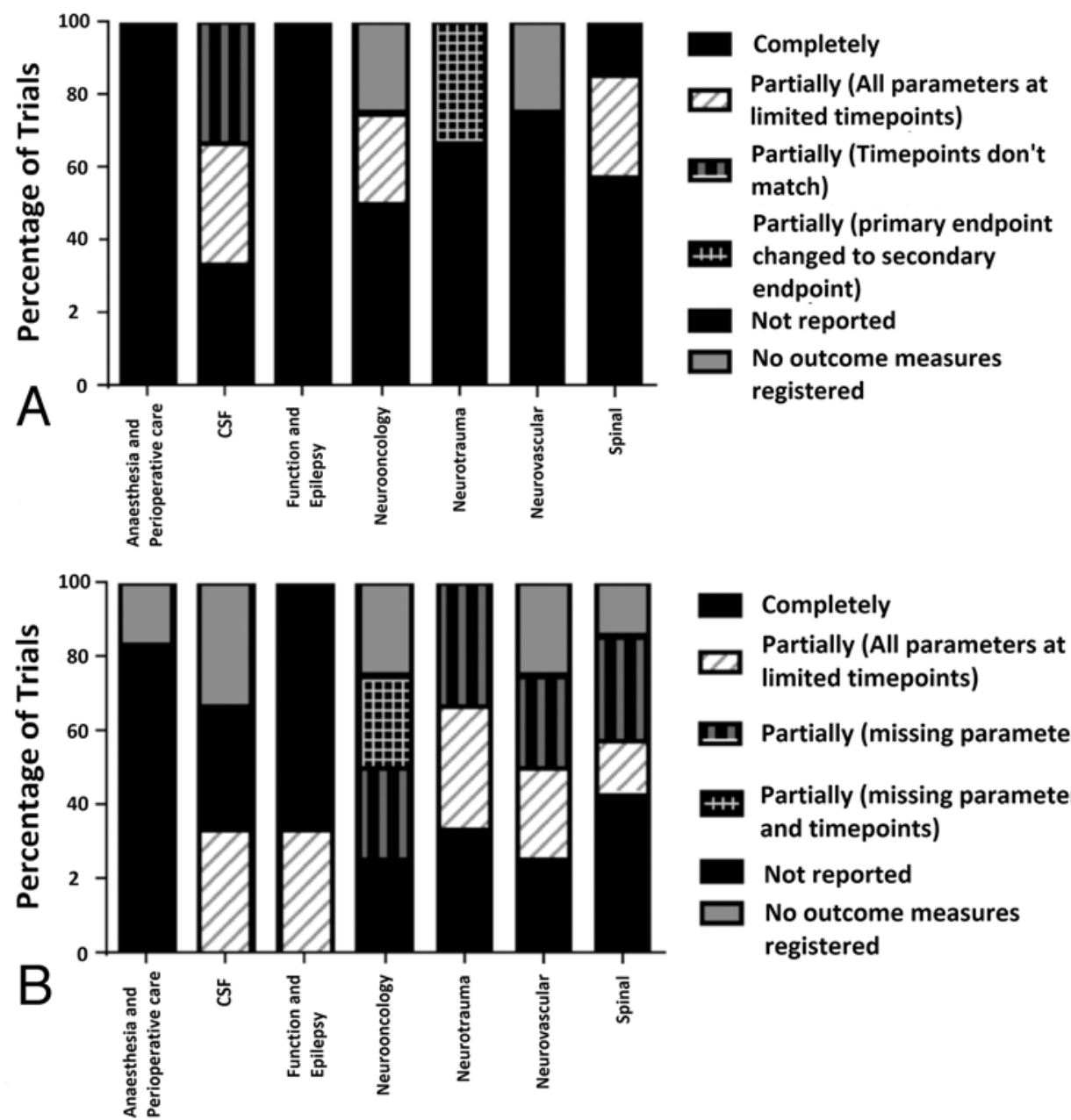

프 Partially (missing parameters)

\# Partially (missing parameters and timepoints)

Not reported

$\square$ No outcome measures registered

FIG. 4. Chart showing the quality of (A) primary outcome measure and (B) secondary outcome measure reporting by neurosurgical subspecialty.

were more aligned with the study of Chapman et al.; our finding of a $26.6 \%$ rate of discontinued neurosurgical trials was higher than that of the general surgical cohort in the Chapman et al. study. The differences in the assessed interventions between the studies may explain the higher neurosurgical trial discontinuation rate. Within our study, pharmacological interventions were assessed in a third of the trials compared with almost double (63\%) those in the Chapman et al. study. Although neither our study nor Chapman's study identified significant differences in discontinuation rates between these cohorts, a larger study investigating $863 \mathrm{RCTs}$ found that trials assessing invasive treatments had higher discontinuation rates than those assessing noninvasive treatments. ${ }^{18}$

There is a wide range of reasons for why a clinical trial may be discontinued early, ranging from unexpected harm, to futility, to recruitment or funding issues. The premature termination of a clinical trial may also be related to prespecified criteria and should not always be considered a failure. The most commonly cited reason for discontinuation of neurosurgical RCTs was slow or insufficient enrolment. The importance of slow or insufficient enrolment is not a problem limited to trials in neurosurgery and a number of other studies have found it to be the primary reason for early disconuation. ${ }^{6,12,18}$ Poor recruitment, although still a major source of discontinuation in medical trials, appears to be less pronounced. Rosenthal et al. found that surgical trials were significantly more likely to be discontinued due to poor recruitment compared with medical trials. ${ }^{18}$ Our study had the highest percentage of trial discontinuation due to poor recruitment (57\%) compared with both the Chapman et al. (44\%) and Rosenthal et al. (43\%) studies. ${ }^{6,18}$ This is likely due to a range of causes, including low caseloads in neurosurgery and issues with capacity in acute brain injury that may hinder recruitment. In addition, the potential for significant complications from studied treatments may drive away candidate patients. These suggestions are, in part, reflected in our data whereby the 2 highest subspecialties for trial discontinuation were epilepsy/ functional neurosurgery (50\%) and neurovascular (33\%). Epilepsy and functional neurosurgery are a small subspecialty within neurosurgery and suffer from difficulties surrounding low caseloads, while conversely the acute nature of neurovascular pathologies (aneurysmal subarachnoid hemorrhage and malignant middle cerebral artery syndrome) may pose obstacles to recruitment. These are important findings as they highlight some of the challenges facing the difficulties for neurosurgical trialists. Without a 
doubt, collaboration (both at the national and international level) is vital to ensure that the issues of poor recruitment can be tackled in a meaningful and effective manner.

In our cohort of discontinued trials, we found that 5 (29.4\%) had provided results; 2 of these studies were published in peer-reviewed articles and a further 3 uploaded results to the clinicaltrials.gov database. This gives our discontinued trials a nonpublication rate of $88 \%$, which is significantly higher than other studies. Kasenda and colleagues found a nonpublication rate of $33.6 \%$ in a cohort of discontinued trials. ${ }^{12}$ Rosenthal et al. found a nonpublication rate of $45 \%$ among their discontinued RCTs. ${ }^{18}$ Our higher nonpublication rate can be partly explained by our shorter follow-up time compared with the other 2 studies, both of which focused on a limited timeframe (2000-2003). Nonetheless, the high nonpublication rate of discontinued trials is equally concerning as data from these studies could affect decision-making for patient care. One approach to address this data loss is the introduction of patient registries that provide continuous data collection and monitoring. This has been achieved with good effect for devices such as shunts for hydrocephalus. The United Kingdom Shunt Registry was established in 1995 and captures a range of data points on shunt insertion, including infection rates. The registry provided strong supportive evidence for the value of antibiotic-impregnated catheters in reducing infection rates compared with conventional catheters. ${ }^{16}$ The success of the registries has led to proposals for the expansion of their use within neurosurgery, including for implants such as cranioplasties..$^{15}$

Compared with the discontinued trials, we found that completed trials had a lower nonpublication rate $(30 \%)$. The nonpublication rate for neurosurgical RCTs was therefore higher compared with the studies of Rosenthal et al. (24\%) and Chapman et al. (27\%). ${ }^{6,18}$ The difference is small and, as previously mentioned, the longer follow-up times are a likely contributor to these differences. Similar to previous studies, we found that discontinued trials had a higher nonpublication rate compared with completed trials. ${ }^{12,18}$ Interestingly, we found no difference in nonpublication rate when we compared studies funded by industry with those that were not. A number of studies have identified industry funding to be associated with nonpublication. Jones and colleagues investigated large RCTs (500 participants or more) and found that industry-funded trials had a nonpublication rate of $32 \%$ compared with only $18 \%$ in nonindustry-funded trials $(\mathrm{p}=0.003) .{ }^{11}$

Within this study, we were also interested in the quality of outcome reporting in the published articles and how this compared with the registered outcomes on clinicaltrials. gov. The CONSORT Group has produced a statement recommending a minimum amount of data that should be reported by RCTs. ${ }^{2}$ With respect to neurosurgery, the CONSORT reporting standards have been recently assessed by Kiehna et al. who concluded that RCT reporting in neurosurgery remains suboptimal. ${ }^{13}$ Our study demonstrated that outcome reporting in neurosurgical RCTs leaves much to be desired, with only $61 \%$ of trials completely reporting primary outcome measures and even lower percentages of complete secondary outcome measure reporting at $33.3 \%$. These findings, however, compare favorably those of a study in a psychology journal evaluating outcome reporting that identified 39 registered trials, only 2 of which predefined outcome measures and only 1 of which had completed outcome reporting at publication. ${ }^{1}$ Incomplete outcome reporting has been quantified in detail by Chan et al. who found that in a cohort of 102 trials, $50 \%$ of efficacy and $45 \%$ of harm outcomes were reported completely. ${ }^{5}$ They also showed that statistically significant outcomes were more likely to be published than nonsignificant outcomes.

The selection of studies for inclusion in this work from a trial registration database means the data loss quantified here is almost certainly an underestimate because of problems that still exist regarding the registration of clinical trials. Killeen et al., in a recent analysis of trial registration in surgical journals, reported a failure to register trials in $34.9 \%$ of cases (86 trials) and "inadequate" registration in $21 \%$ of cases (52 trials). ${ }^{14}$ However, the calculated publication rate of this cohort of studies will likely increase with time as completed trials toward the end of the investigated period will not have had sufficient time to publish results within 2.5 years before our assessment. Support for this is evidenced by the time it took some studies to be published. An additional limitation was that while the inclusion criteria were defined using registry dates from clinicaltrials. gov, there was no way to match the inclusion period to a PubMed searchable parameter. This meant it was not possible to obtain information on published trials that should have been registered in the study period but were not. The number of published but unregistered trials was therefore not obtainable.

\section{Conclusions}

In this study, we have provided the most comprehensive assessment to date of neurosurgical trial discontinuation and publication outcome. Our analysis highlights that more than one-fifth of neurosurgical RCTs are discontinued early and almost a third of those that are completed remain unpublished. These findings are consistent with studies investigating surgical RCTs. However, we found that poor recruitment was a disproportionately major driving force for the early discontinuation of neurosurgical trials. This finding highlights the importance of trialists identifying strategies for improving patient recruitment.

\section{Acknowledgments}

We would like to extend our thanks to the PIs who took the time to respond to our querying email.

Aimun A. B. Jamjoom is currently undertaking a $\mathrm{PhD}$ funded by the Wellcome Trust; however, the work in this manuscript was not directly supported by this grant.

\section{References}

1. Azar M, Riehm KE, McKay D, Thombs BD: Transparency of outcome reporting and trial registration of randomized controlled trials published in the Journal of Consulting and Clinical Psychology. PLoS One 10:e0142894, 2015

2. Begg C, Cho M, Eastwood S, Horton R, Moher D, Olkin I, et al: Improving the quality of reporting of randomized controlled trials. The CONSORT statement. JAMA 276:637639, 1996 
3. Booth CM, Tannock IF: Randomised controlled trials and population-based observational research: partners in the evolution of medical evidence. Br J Cancer 110:551-555, 2014

4. Chalmers I, Glasziou P, Godlee F: All trials must be registered and the results published. BMJ 346:f 105, 2013

5. Chan AW, Hróbjartsson A, Haahr MT, Gøtzsche PC, Altman DG: Empirical evidence for selective reporting of outcomes in randomized trials: comparison of protocols to published articles. JAMA 291:2457-2465, 2004

6. Chapman SJ, Shelton B, Mahmood H, Fitzgerald JE, Harrison EM, Bhangu A: Discontinuation and non-publication of surgical randomised controlled trials: observational study. BMJ 349:g6870, 2014

7. Dekkers W, Boer G: Sham neurosurgery in patients with Parkinson's disease: is it morally acceptable? J Med Ethics 27:151-156, 2001

8. Dwan K, Gamble C, Williamson PR, Kirkham JJ: Systematic review of the empirical evidence of study publication bias and outcome reporting bias - an updated review. PLoS One 8:e66844, 2013

9. Gagne JJ, Thompson L, O'Keefe K, Kesselheim AS: Innovative research methods for studying treatments for rare diseases: methodological review. BMJ 349:g6802, 2014

10. Ioannidis JPA: Clinical trials: what a waste. BMJ 349:g7089, 2014

11. Jones CW, Handler L, Crowell KE, Keil LG, Weaver MA, Platts-Mills TF: Non-publication of large randomized clinical trials: cross sectional analysis. BMJ 347:f6104, 2013

12. Kasenda B, von Elm E, You J, Blümle A, Tomonaga Y, Saccilotto R, et al: Prevalence, characteristics, and publication of discontinued randomized trials. JAMA 311:1045-1051, 2014

13. Kiehna EN, Starke RM, Pouratian N, Dumont AS: Standards for reporting randomized controlled trials in neurosurgery. $\mathbf{J}$ Neurosurg 114:280-285, 2011

14. Killeen S, Sourallous P, Hunter IA, Hartley JE, Grady HL: Registration rates, adequacy of registration, and a comparison of registered and published primary outcomes in randomized controlled trials published in surgery journals. Ann Surg 259:193-196, 2014
15. Kolias AG, Bulters DO, Cowie CJ, Wilson MH, Afshari FT, Helmy A, et al: Proposal for establishment of the UK Cranial Reconstruction Registry (UKCRR). Br J Neurosurg 28:310 314, 2014

16. Pickard JD, Richards HK, Seeley HM: Impact of antibioticimpregnated catheters on the reduction in operations for cerebrospinal fluid shunt infection since 1995: evidence from the UK Shunt Registry. Fluids Barriers CNS 12 (Suppl 1):O9, 2015 (Abstract)

17. Pollock BE (ed): Guiding Neurosurgery by Evidence. Basel: Karger, 2006

18. Rosenthal R, Kasenda B, Dell-Kuster S, von Elm E, You J, Blümle A, et al: Completion and publication rates of randomized controlled trials in surgery: an empirical study. Ann Surg 262:68-73, 2015

\section{Disclosures}

The authors report no conflict of interest concerning the materials or methods used in this study or the findings specified in this paper.

\section{Author Contributions}

Conception and design: Jamjoom, Demetriades. Acquisition of data: Jamjoom, Gane. Analysis and interpretation of data: Jamjoom, Gane. Drafting the article: Jamjoom, Gane. Critically revising the article: all authors. Reviewed submitted version of manuscript: all authors. Approved the final version of the manuscript on behalf of all authors: Jamjoom. Statistical analysis: Jamjoom, Gane. Study supervision: Demetriades.

\section{Correspondence}

Aimun A. B. Jamjoom, Division of Neurosurgery, Department of Clinical Neurosciences, Western General Hospital, Edinburgh EH4 2XU, United Kingdom. email: aabjamjoom@gmail.com. 